\title{
Sneutrino brane inflation and leptogenesis
}

\author{
M. C. Bento, , 因 R. González Felipe,, , and N. M. C. Santos ${ }^{1,}$ 团 \\ ${ }^{1}$ Departamento de Fúsica and Centro de Física das Interacções Fundamentais, \\ Instituto Superior Técnico, Av. Rovisco Pais, 1049-001 Lisboa, Portugal
}

\begin{abstract}
Modifications to the Friedmann equation in brane cosmology can have important implications for early universe phenomena such as inflation and the generation of the baryon asymmetry. We study a simple scenario of chaotic brane inflation where, in a minimal supersymmetric seesaw model, the scalar superpartner of a heavy singlet Majorana neutrino drives inflation and, simultaneously, generates the required lepton asymmetry through its direct out-of-equilibrium decays after the inflationary era. For a gravitino mass in the range $m_{3 / 2} \simeq 100 \mathrm{GeV}-1 \mathrm{TeV}$, we find that successful nucleosynthesis and leptogenesis in this framework require that the 5D Planck mass is in the range $M_{5} \simeq 10^{10}-10^{13} \mathrm{GeV}$ and the reheating temperature $T_{r h} \simeq 10^{6}-10^{8} \mathrm{GeV}$.

PACS numbers: 98.80.Cq, 98.80.Es, 04.50.+h
\end{abstract}

\section{INTRODUCTION}

Today there is a wide consensus that the early universe underwent a period of cosmological inflation [1]. Inflationary era can be regarded as a necessary stage, responsible not only for the observed flatness, homogeneity and isotropy of the present universe, but also for the origin of the density fluctuations as observed by the Cosmic Background Explorer (COBE) and, more recently, the Wilkinson Microwave Anisotropy Probe (WMAP) satellites 2]. At the end of inflation, the universe was in a cold and low-entropy state and it must has been subsequently reheated to become a high-entropy and radiationdominated universe. Such a reheating process could occur, for instance, through the coherent oscillations of the inflaton field about the minimum of the potential until the age of the universe equals the lifetime of the inflaton. The latter decays into ordinary particles, which then scatter and thermalize. Besides entropy creation, the right abundance of baryons must be created after the inflationary epoch. This usually poses serious problems in constructing particle physics models which lead simultaneously to a successful inflationary and baryogenesis scenario. In particular, the reheating temperature is typically too low when compared with the grand unification scale, at which baryogenesis is expected to take place in the simplest GUTs. Moreover, any preexisting baryon asymmetry would be erased by the anomalous sphaleron processes [3] unless an initial $B-L$ asymmetry is generated.

Another major obstacle in constructing viable supergravity-inspired cosmological models is the overproduction of gravitinos. In conventional scenarios, the gravitino mass is expected to be comparable to the masses of the supersymmetric partners of the standard model particles and, therefore, $m_{3 / 2} \lesssim \mathrm{a}$ few $\mathrm{TeV}$ in order to solve the

\footnotetext{
*Electronic address: bento@sirius.ist.utl.pt

${ }^{\dagger}$ Electronic address: gonzalez@cfif.ist.utl.pt

‡Electronic address: ncsantos@cfif.ist.utl.pt
}

gauge hierarchy problem. Since the gravitino coupling to matter is suppressed by the Planck mass $M_{P}$, its lifetime is $\tau_{3 / 2} \sim M_{P}^{2} / m_{3 / 2}^{3} \sim 10^{8}\left(100 \mathrm{GeV} / m_{3 / 2}\right)^{3}$ s. During the reheating phase gravitinos can be thermally produced through scatterings in the plasma. However, if they are overproduced after inflation, their decay products could put at risk the successful predictions of primordial nucleosynthesis [4, 5]. Since in standard cosmology their abundance is proportional to the reheating temperature, $T_{r h}$, constraints from big bang nucleosynthesis (BBN) yield a stringent upper bound on the allowed $T_{r h}$ after inflation: $T_{r h} \lesssim 10^{7}-10^{10} \mathrm{GeV}$ for $100 \mathrm{GeV} \lesssim m_{3 / 2} \lesssim 1 \mathrm{TeV}[5]$.

Among the current chaotic inflationary scenarios in supersymmetric seesaw theories [6, 7, 8], inflation driven by the scalar superpartner of the right-handed Majorana neutrino is one of the simplest and most economical ones. In this context, the heavy singlet neutrinos are naturally invoked to give masses to the light neutrinos through the seesaw mechanism [9]. Moreover, their superpartners the sneutrino fields - can play the role of the inflaton. Also, if $C P$ is violated the sneutrino decays will create a lepton asymmetry, which is then converted into a baryon asymmetry by the electroweak sphalerons. This is indeed an appealing scenario, since cosmology and particle physics merge together to make predictions about the early universe and the low-energy physics that we test today.

There is, however, a drawback in the above-mentioned framework. In the usual chaotic inflation scenario based on standard cosmology, super-Planckian inflaton field values $\sim 3 M_{P}$ are typically required to allow for a sufficiently long period of inflation (the so-called $\eta$ problem). Thus one expects nonrenormalizable quantum corrections of the order of $\mathcal{O}\left[\left(\phi / M_{P}\right)^{n}\right]$ (with $n>4$ ) to destroy the flatness of the potential necessary for successful inflation. A possible way out of this situation is to consider, for instance, higher-dimensional cosmological models, where our four-dimensional world is viewed as a 3-brane embedded in a higher-dimensional bulk.

A remarkable feature of brane cosmology is the modification of the expansion rate of the universe $H$ before the nucleosynthesis era [10]. While in standard cosmol- 
ogy the expansion rate scales with the energy density $\rho$ as $H \propto \sqrt{\rho}$, this dependence becomes $H \propto \rho$ at very high energies in brane cosmology. This behavior, which appears to be quite generic and not specific to RandallSundrum braneworld scenarios [11], may have drastic consequences on early universe phenomena such as inflation and the generation of the baryon asymmetry. In particular, modifications to the Friedmann equation not only ease the conditions for slow-roll inflation but also enable the simplest chaotic inflation models to inflate at field values far below $M_{P}$, thus avoiding well-known difficulties with higher-order nonrenormalizable terms. Another important difference between standard and brane cosmologies is in the predictions for gravitino production. For a given value of the brane tension or, equivalently, of the $5 \mathrm{D}$ Planck mass, $M_{5}$, the gravitino abundance in the brane decreases as $T_{r h}$ increases. Therefore, in contrast to standard cosmology, BBN constraints in the brane scenario imply a lower (rather than an upper) bound on the reheating temperature.

The aim of this paper is combines the above ideas, i.e. chaotic inflation and direct leptogenesis through sneutrino decays in the braneworld context 12]. More precisely, we study a simple scenario of chaotic brane inflation where, in a minimal supersymmetric seesaw model, the scalar superpartner of a heavy singlet Majorana neutrino drives inflation and, simultaneously, generates the required lepton asymmetry through its direct out-ofequilibrium decays after the inflationary era. This requires the reheating temperature $T_{r h}$ to be smaller than the sneutrino inflaton mass, $M_{1}$. In this framework, there exists a direct connection between the brane inflationary era, the reheating of the universe, leptogenesis from sneutrino decays and the light neutrino properties, which allows us to strongly constrain the fundamental $5 \mathrm{D}$ Planck mass and the reheating temperature of the universe.

We shall not consider here the case where leptogenesis is purely thermal, i.e. when $T_{r h}>M_{1}$. In this case, any lepton asymmetry generated through the sneutrino inflaton decays is erased by thermal effects, and therefore, leptogenesis is driven by the out-of-equilibrium decays of the heavy singlet Majorana neutrinos and sneutrinos thermally created.

\section{SNEUTRINO BRANEWORLD INFLATION}

We consider the simplest scenario where heavy righthanded neutrinos $N_{i}, i=1,2,3$, with masses $M_{i}$, are added to the usual particle content of the minimal supersymmetric standard model. In what follows we assume that the seesaw mechanism [9] is operative in the brane scenario and gives masses to the light neutrinos. We remark however that in the presence of extra dimensions other mechanisms are viable as well. In particular, it is possible to generate light neutrino masses without the need for a superheavy mass scale [13, 14]. We also assume that the lightest right-handed sneutrino $\widetilde{N}_{1}$ acts as an inflaton with a potential simply given by the mass term

$$
V=\frac{1}{2} M_{1}^{2} \widetilde{N}_{1}^{2} .
$$

For simplicity we neglect the dynamics of the heavier sneutrinos $\widetilde{N}_{2,3}$. Under these assumptions, the model reduces to the simplest braneworld chaotic inflation scenario with a quadratic potential of the form $V=\frac{1}{2} m^{2} \phi^{2}$.

We start by reviewing the constraints resulting from WMAP bounds on inflationary observables. In standard cosmology, the requirement that perturbations have the observed amplitude fixes the inflaton mass: $m \simeq$ $10^{13} \mathrm{GeV}$. However, due to the presence of the brane tension, $\lambda$, the Friedmann equation in the braneworld context receives an additional term quadratic in the density [10],

$$
H^{2}=\frac{8 \pi}{3 M_{P}^{2}} \rho\left(1+\frac{\rho}{2 \lambda}\right)
$$

where $M_{P}=1.22 \times 10^{19} \mathrm{GeV}$ is the $4 \mathrm{D}$ Planck mass. In the above expression for $H$, we have set the fourdimensional cosmological constant to zero and assumed that inflation rapidly makes any dark radiation term negligible. Notice that Eq. (2) reduces to the usual Friedmann equation at sufficiently low energies, $\rho \ll \lambda$. The brane tension relates the Planck mass in four and five dimensions via

$$
M_{P}=\sqrt{\frac{3}{4 \pi}} \frac{M_{5}^{3}}{\sqrt{\lambda}} .
$$

Successful big bang nucleosynthesis requires that the change in the expansion rate due to the new terms in the Friedmann equation be sufficiently small at scales $\sim \mathcal{O}(\mathrm{MeV})$. This implies the lower bound $\lambda \gtrsim(1 \mathrm{MeV})^{4}$, or using Eq. (3), $M_{5} \gtrsim 40 \mathrm{TeV}$ [15]. A more stringent bound, $M_{5} \gtrsim 10^{5} \mathrm{TeV}$, can be obtained by requiring the theory to reduce to Newtonian gravity on scales larger than $1 \mathrm{~mm} \mathrm{[16]}$.

In the slow-roll approximation, the total number of $e$ folds during inflation is given by 16 ]

$$
N \simeq-\frac{8 \pi}{M_{P}^{2}} \int_{\phi_{i}}^{\phi_{f}} \frac{V}{V^{\prime}}\left(1+\frac{V}{2 \lambda}\right) d \phi
$$

where $\phi_{i}$ and $\phi_{f}$ are the values of the scalar field at the beginning and at the end of the expansion, respectively. The value $\phi_{f}$ can be computed from the condition $\max \left\{\epsilon\left(\phi_{f}\right),\left|\eta\left(\phi_{f}\right)\right|\right\}=1$, where $\epsilon$ and $\eta$ are the slow-roll parameters, given by

$$
\begin{aligned}
\epsilon & \equiv \frac{M_{P}^{2}}{16 \pi}\left(\frac{V^{\prime}}{V}\right)^{2} \frac{1+V / \lambda}{(1+V / 2 \lambda)^{2}}, \\
\eta & \equiv \frac{M_{P}^{2}}{8 \pi} \frac{V^{\prime \prime}}{V} \frac{1}{1+V / 2 \lambda} .
\end{aligned}
$$


Other key parameters during inflation are the spectra of scalar [16] and tensor [17] perturbations at the Hubble radius crossing:

$$
\begin{aligned}
& A_{s}^{2}=\frac{512 \pi}{75 M_{P}^{6}} \frac{V^{3}}{V^{\prime 2}}\left(1+\frac{V}{2 \lambda}\right)^{3}, \\
& A_{t}^{2}=\frac{32}{75 M_{P}^{4}} V\left(1+\frac{V}{2 \lambda}\right) F^{2} .
\end{aligned}
$$

Here

$$
\begin{aligned}
F^{2}(x) & =\left[\sqrt{1+x^{2}}-x^{2} \sinh ^{-1} \frac{1}{x}\right]^{-1}, \\
x & =\left[\frac{2 V}{\lambda}\left(1+\frac{V}{2 \lambda}\right)\right]^{1 / 2} .
\end{aligned}
$$

In the low-energy limit, when $V \ll \lambda$ (i.e. $x \ll 1$ ), one has $F^{2} \approx 1$, whereas in the high-energy limit, $V \gg \lambda$, we obtain $F^{2} \approx 3 V / 2 \lambda$.

The scale dependence of the scalar perturbations is described by the spectral tilt

$$
n_{s}-1 \equiv \frac{d \ln A_{s}^{2}}{d \ln k} \simeq-6 \epsilon+2 \eta,
$$

and its running is given by

$$
\alpha_{s} \equiv \frac{d n_{s}}{d \ln k} \simeq 16 \epsilon \eta-18 \epsilon^{2}-2 \xi
$$

where

$$
\xi \equiv \frac{M_{P}^{4}}{(8 \pi)^{2}} \frac{V^{\prime} V^{\prime \prime \prime}}{V^{2}} \frac{1}{(1+V / 2 \lambda)^{2}} .
$$

Finally, the tensor power spectrum amplitude can be parametrized by the tensor-to-scalar ratio

$$
r_{s} \equiv 16 \frac{A_{t}^{2}}{A_{s}^{2}}
$$

which is consistent with the normalization of Ref. [18] in the low-energy limit.

For the simple quadratic potential of Eq. (1) one gets in the high-energy approximation ${ }^{1}$

$$
\begin{aligned}
n_{s} & \simeq \frac{2\left(N_{\star}-2\right)}{2 N_{\star}+1}, \\
\alpha_{s} & \simeq-\frac{10}{\left(2 N_{\star}+1\right)^{2}}, \\
r_{s} & \simeq \frac{24}{2 N_{\star}+1},
\end{aligned}
$$

\footnotetext{
1 Our expressions do not coincide with the ones of Ref. 19 because we take the condition $\epsilon=1$ rather than $\eta=1$ to find $\phi_{f}$. The difference in the numerical values is however insignificant.
}

where $N_{\star}$ is the number of $e$-folds before the end of inflation at which observable perturbations are generated ${ }^{2}$. Hence, taking e.g. $N_{\star}=60$, we get $n_{s} \approx 0.96, \alpha_{s} \approx$ $-6.83 \times 10^{-4}, r_{s} \approx 0.2$, well within the WMAP bounds on these quantities [18]

$$
0.94 \leq n_{s} \leq 1.01,-0.02 \leq \alpha_{s} \leq 0.02, r_{s} \leq 0.35
$$

at $95 \% \mathrm{CL}$.

Using Eq. (7) the mass parameter $m$ can be expressed in terms of $A_{s}$ and $N_{\star}$ as

$$
m=\left(\frac{75 \sqrt{3} \pi A_{s}^{2}}{8 \sqrt{2}\left(1+2 N_{\star}\right)^{5 / 2}}\right)^{1 / 3} M_{5} .
$$

Inserting into this expression the COBE normalization $A_{s}\left(\phi_{\star}\right) \approx 2 \times 10^{-5}$ and using $N_{\star}=60$, we obtain

$$
m \approx 4.5 \times 10^{-5} M_{5} .
$$

Let us note that the above analysis has been done assuming that the high energy approximation is valid, i.e. $V / \lambda \gg 1$. It is easy to show that this requirement imposes a rather weak constraint on the fundamental scale $M_{5}$, namely, $M_{5} \ll 10^{17} \mathrm{GeV}$. It is also worth remarking that, in contrast to the standard cosmology case, it is possible to obtain sufficient inflation in the braneworld context for sub-Planckian initial values of the inflaton field. Indeed, taking for instance $N=70$, the minimum number of $e$-folds required to solve the initial conditions problems of standard cosmology, one estimates $\phi_{i} \approx 3 \times 10^{2} M_{5}$, which when combined with the above bound on $M_{5}$ implies $\phi_{i}<M_{P}$.

\section{REHEATING AND DIRECT LEPTOGENESIS FROM SNEUTRINO DECAYS}

After inflation, the cold inflaton-dominated universe undergoes a phase of reheating, during which the inflaton decays into normal particles and the universe becomes radiation-dominated. Of particular interest is the reheating temperature, $T_{r h}$, which is defined by assuming an instantaneous conversion of the inflaton energy into radiation, when the decay width of the inflaton, $\Gamma_{\phi}$, equals the expansion rate of the universe, $H$. In the braneworld scenario, $H$ is given by

$$
H \simeq \sqrt{\frac{4 \pi}{3 \lambda}} \frac{\rho}{M_{P}}=\frac{4 \pi}{3} \frac{\rho}{M_{5}^{3}},
$$

in the high energy approximation. If the total inflaton energy density is instantaneously converted into radiation,

\footnotetext{
${ }^{2}$ In standard cosmology, $N_{\star}=55$ is found to be a reasonable fiducial value. This number is expected to be higher in the brane scenario [20]; in Ref. [21] the upper bound $N_{\star}<75$ is found.
} 
then we can identify $\rho=\rho_{R}=\left(\pi^{2} / 30\right) g_{*} T^{4}$, where $g_{*}$ is the effective number of relativistic degrees of freedom at the temperature $T$. In particular, $g_{*}=915 / 4=228.75$ in the MSSM for temperatures above the SUSY breaking scale $\sim \mathcal{O}(\mathrm{TeV})$. Thus we obtain

$$
H \simeq \frac{2 \pi^{3} g_{*}}{45} \frac{T^{4}}{M_{5}^{3}} .
$$

The condition $H\left(T_{r h}\right)=\Gamma_{\phi}$ leads then to the relation

$$
T_{r h}=\left(\frac{45}{2 \pi^{3} g_{*}} \Gamma_{\phi} M_{5}^{3}\right)^{1 / 4} .
$$

In the sneutrino inflation scenario we are considering, $\phi \equiv \tilde{N}_{1}, m \equiv M_{1}$ and

$$
\Gamma_{\phi} \equiv \Gamma_{N_{1}}=\frac{1}{4 \pi}\left(Y_{\nu} Y_{\nu}^{\dagger}\right)_{11} M_{1}
$$

where $Y_{\nu}$ is the Dirac neutrino Yukawa matrix. Substituting Eq. (24) into (23), we get

$$
T_{r h}=\left[\frac{45}{8 \pi^{4} g_{*}}\left(Y_{\nu} Y_{\nu}^{\dagger}\right)_{11} M_{1} M_{5}^{3}\right]^{1 / 4} .
$$

At the end of inflation, when the Hubble parameter becomes smaller than $M_{1}$, the inflaton field $\widetilde{N}_{1}$ begins to oscillate coherently around the minimum of the potential. If $C P$ is not conserved, the decays of $\widetilde{N}_{1}$ into leptons, Higgs and the corresponding antiparticles can produce a net lepton asymmetry. We require such decays to occur out of equilibrium, i.e. $T_{r h}<M_{1}$, so that leptogenesis is driven by the decays of cold sneutrino inflatons and the produced lepton asymmetry is not washed out by lepton-number violating interactions mediated by $N_{1}$. The above requirement, combined with Eqs. (25) and (20), leads to the following constraint on the Dirac neutrino Yukawa couplings

$$
\left(Y_{\nu} Y_{\nu}^{\dagger}\right)_{11} \lesssim 3.5 \times 10^{-10},
$$

which is independent of the fundamental scale $M_{5}$. Therefore, as in the standard sneutrino inflationary scenario [8], the quantity $\left(Y_{\nu} Y_{\nu}^{\dagger}\right)_{11}$ is required to be very small.

The lepton number density generated by the sneutrino condensate is given by

$$
n_{L}=\epsilon_{1} \frac{\rho}{M_{1}},
$$

where the parameter $\epsilon_{1}$ denotes the $C P$ asymmetry in the $\widetilde{N}_{1}$ decays. Recalling that the entropy density is $s=\left(2 \pi^{2} g_{*} / 45\right) T^{3}$, the lepton-to-entropy ratio can be written as $[6,7]$

$$
Y_{L} \equiv \frac{n_{L}}{s}=\frac{3}{4} \epsilon_{1} \frac{T_{r h}}{M_{1}},
$$

with $T_{r h}$ given by Eq. (25).
Assuming the mass hierarchy $M_{1} \ll M_{2}, M_{3}$ for the heavy Majorana neutrinos, one has [7]

$$
\epsilon_{1} \simeq \frac{3}{8 \pi} \frac{M_{1}}{v^{2} \sin ^{2} \beta} \frac{\operatorname{Im}\left[Y_{\nu} \mathcal{M}_{\nu}^{*} Y_{\nu}^{T}\right]_{11}}{\left(Y_{\nu} Y_{\nu}^{\dagger}\right)_{11}},
$$

where $\mathcal{M}_{\nu}$ is the light neutrino effective mass matrix, $v \simeq 174 \mathrm{GeV}$ and $\tan \beta$ is the ratio of the vacuum expectation values of the two Higgs doublets of the MSSM. It is convenient to parametrize the above expression in the form

$$
\epsilon_{1}=\epsilon_{1}^{\max } \sin \delta_{L},
$$

where $\delta_{L}$ is an effective leptogenesis phase and $\epsilon_{1}^{\max }$ is the maximal asymmetry. In particular, for hierarchical light neutrinos with $m_{1} \simeq 0 \ll m_{2} \simeq \sqrt{\Delta m_{\text {sol }}^{2}} \ll m_{3} \simeq$ $\sqrt{\Delta m_{\text {atm }}^{2}}$, one obtains ${ }^{3}[22]$

$$
\epsilon_{1}^{\max }=\frac{3}{8 \pi} \frac{M_{1} \sqrt{\Delta m_{\mathrm{atm}}^{2}}}{v^{2} \sin ^{2} \beta},
$$

where the squared mass differences, measured in solar and atmospheric neutrino oscillation experiments, are $\Delta m_{\mathrm{sol}}^{2} \simeq 7.1 \times 10^{-5} \mathrm{eV}^{2}$ and $\Delta m_{\mathrm{atm}}^{2} \simeq 2.6 \times 10^{-3} \mathrm{eV}^{2}$. With $\sin \beta \simeq 1$ (large $\tan \beta$ regime) the maximal $C P$ asymmetry is then given by

$$
\epsilon_{1}^{\max } \simeq 2 \times 10^{-10}\left(\frac{M_{1}}{10^{6} \mathrm{GeV}}\right) .
$$

The lepton asymmetry produced before the electroweak phase transition is partially converted into a baryon asymmetry via the sphaleron effects, [3]

$$
Y_{B} \equiv \frac{n_{B}}{\mathrm{~s}}=\xi Y_{L},
$$

where $\xi=-8 / 23$ for the MSSM 24].

From Eqs. (28), (30), (32) and (33), we obtain

$$
Y_{B}=5.3 \times 10^{-11} \sin \delta_{L}\left(\frac{T_{r h}}{10^{6} \mathrm{GeV}}\right) .
$$

From the observational side, WMAP bounds on the baryon-to-photon ratio, $\eta_{B} \equiv n_{B} / n_{\gamma}$, imply [2]

$$
\eta_{B}=6.1_{-0.2}^{+0.3} \times 10^{-10} .
$$

Recalling that $s \simeq 7.04 n_{\gamma}$, Eqs. (34) and (35) lead to

$$
T_{r h}=\frac{1.6 \times 10^{6}}{\left|\sin \delta_{L}\right|} \mathrm{GeV},
$$

which yields the following lower bound on the reheating temperature

$$
T_{r h} \gtrsim 1.6 \times 10^{6} \mathrm{GeV},
$$

for $\epsilon_{1}=\epsilon_{1}^{\max }$.

\footnotetext{
${ }^{3}$ For a more accurate bound and a detailed discussion see e.g. Ref. [23].
} 


\section{CONSTRAINTS ON GRAVITINO PRODUCTION}

As discussed in the Introduction, a viable cosmological supergravity-inspired scenario has to avoid the so-called gravitino problem, i.e. for unstable gravitinos, their decay products should not alter the BBN predictions for the abundance of light elements in the universe. This requirement is usually translated into an upper bound on the gravitino abundance 5]:

$$
\eta_{3 / 2} \equiv \frac{n_{3 / 2}}{n_{\gamma}} \lesssim \frac{\zeta_{\max }}{m_{3 / 2}}
$$

where $m_{3 / 2}$ is the gravitino mass, $n_{\gamma}(T)=2 \zeta(3) T^{3} / \pi^{2}$ is the photon density and $\zeta_{\max }$ is a parameter that accounts for the maximum gravitino abundance allowed by the BBN predictions. In particular, for $m_{3 / 2} \simeq 100 \mathrm{GeV}$ and a gravitino lifetime $\tau_{3 / 2} \simeq 10^{8} \mathrm{~s}$, one has $[\underline{5}$ ]

$$
\zeta_{\max } \simeq 5 \times 10^{-12} \mathrm{GeV}
$$

while for $m_{3 / 2} \simeq 1 \mathrm{TeV}$ and $\tau_{3 / 2} \simeq 10^{5} \mathrm{~s}$,

$$
\zeta_{\max } \simeq 10^{-8} \mathrm{GeV} \text {. }
$$

The gravitino abundance at a given temperature $T<$ $T_{r h}$ can be obtained from the Boltzmann equation

$$
\frac{d n_{3 / 2}}{d t}+3 H n_{3 / 2}=C_{3 / 2}(T),
$$

where $C_{3 / 2}(T)$ is the collision term. Assuming constant entropy, the integration of Eq. (41) yields

$$
\eta_{3 / 2}(T)=\frac{g_{*}(T)}{g_{*}\left(T_{r h}\right)} \frac{C_{3 / 2}\left(T_{r h}\right)}{H\left(T_{r h}\right) n_{\gamma}\left(T_{r h}\right)},
$$

where $g_{*}(T)=43 / 11 \simeq 3.91$ for $T<1 \mathrm{MeV}$, whereas $g_{*}\left(T_{r h}\right)=228.75$. The collision term is given by [25]

$$
C_{3 / 2}(T) \simeq \alpha(T)\left(1+\beta(T) \frac{m_{\tilde{g}^{2}}}{m_{3 / 2}^{2}}\right) \frac{T^{6}}{M_{P}^{2}},
$$

where $m_{\tilde{g}}$ is the low-energy gluino mass; $\alpha(T)$ and $\beta(T)$ are slowly-varying functions of the temperature. For $T_{r h} \sim 10^{6} \mathrm{GeV}$ (cf. Eq. (37)) we estimate $\alpha\left(T_{r h}\right) \simeq 2.38$ and $\beta\left(T_{r h}\right) \simeq 0.13$.

Substituting Eqs. (22) and (43) into Eq. (42), we obtain for the gravitino abundance

$$
\eta_{3 / 2} \simeq 5.3 \times 10^{-4}\left(1+0.13 \frac{m_{\tilde{g}^{2}}}{m_{3 / 2}^{2}}\right) \frac{M_{5}^{3}}{T_{r h} M_{P}^{2}} .
$$

Taking into account the BBN gravitino abundance bound (see Eqs. (38)-(40)) we find

$$
\begin{aligned}
& T_{r h} \gtrsim 970\left(\frac{M_{5}}{10^{10} \mathrm{GeV}}\right)^{3} \text { for } m_{3 / 2}=100 \mathrm{GeV}, \\
& T_{r h} \gtrsim 0.4\left(\frac{M_{5}}{10^{10} \mathrm{GeV}}\right)^{3} \quad \text { for } m_{3 / 2}=1 \mathrm{TeV}, \quad
\end{aligned}
$$

for a typical gluino mass of $m_{\tilde{g}}=1 \mathrm{TeV}$. We notice that in contrast with the standard cosmology case, where the gravitino production imposes an upper bound on the reheating temperature of the universe, the BBN constraints in the braneworld scenario imply a lower bound on $T_{r h}$ instead.

Combining the requirement to avoid thermal leptogenesis, $T_{r h}<M_{1}$, with Eqs. (20) and (45), we get

$$
\begin{array}{ll}
M_{5} \lesssim 2.1 \times 10^{11} \mathrm{GeV} & \text { for } m_{3 / 2}=100 \mathrm{GeV} \\
M_{5} \lesssim 1.1 \times 10^{13} \mathrm{GeV} & \text { for } m_{3 / 2}=1 \mathrm{TeV}
\end{array}
$$

We note that these bounds are more stringent than the one imposed by the validity of the high energy approximation $\left(M_{5} \lesssim 10^{17} \mathrm{GeV}\right)$. The above limits can also be translated into upper bounds on the lightest right-handed Majorana neutrino mass:

$$
\begin{aligned}
& M_{1} \lesssim 9.6 \times 10^{6} \mathrm{GeV} \quad \text { for } m_{3 / 2}=100 \mathrm{GeV}, \\
& M_{1} \lesssim 4.7 \times 10^{8} \mathrm{GeV} \quad \text { for } m_{3 / 2}=1 \mathrm{TeV}
\end{aligned}
$$

One should check whether the above bounds are compatible with the requirement that the contribution of gravitinos to the energy density of the universe does not exceed the observed matter density limit. From the gravitino abundance (44) we can estimate their contribution to the closure density:

$$
\Omega_{3 / 2} h^{2}=m_{3 / 2} \eta_{3 / 2} n_{\gamma 0} h^{2} \rho_{c}^{-1} .
$$

Here $\rho_{c}=3 H_{0}^{2} M_{P}^{2} / 8 \pi=8.07 \times 10^{-47} h^{2} \mathrm{GeV}^{4}$ is the critical density and $n_{\gamma 0}=3.15 \times 10^{-39} \mathrm{GeV}^{3}$ is the photon density. We obtain

$$
\Omega_{3 / 2} h^{2} \simeq 2 \times 10^{-7}\left(\frac{M_{5}}{10^{10} \mathrm{GeV}}\right)^{3}\left(\frac{10^{6} \mathrm{GeV}}{T_{r h}}\right),
$$

for $m_{3 / 2} \simeq 100 \mathrm{GeV}-1 \mathrm{TeV}$. Using the WMAP bound on the matter density of the universe, $\Omega_{m} h^{2}<0.143$ [2], Eq. (49) implies the following relation between $T_{r h}$ and $M_{5}$ :

$$
T_{r h} \gtrsim 1.4\left(\frac{M_{5}}{10^{10} \mathrm{GeV}}\right)^{3} .
$$

Comparing the BBN gravitino production constraint (45) with the bound (50), we see that the latter is more stringent for a gravitino mass of the order of $1 \mathrm{TeV}$.

\section{DISCUSSION AND CONCLUSION}

We now put together the various constraints we have derived sofar. In Figure 1 we show the dependence of the 5D Planck mass $M_{5}$ on the effective leptogenesis phase $\delta_{L}$, for two values of the gravitino mass, $m_{3 / 2}=100 \mathrm{GeV}$ and $1 \mathrm{TeV}$. The lower bound on $M_{5}$ (solid lines) comes from the direct leptogenesis condition, i.e. $T_{r h}<M_{1}$, together with Eqs. (20) and (36). The upper bound 

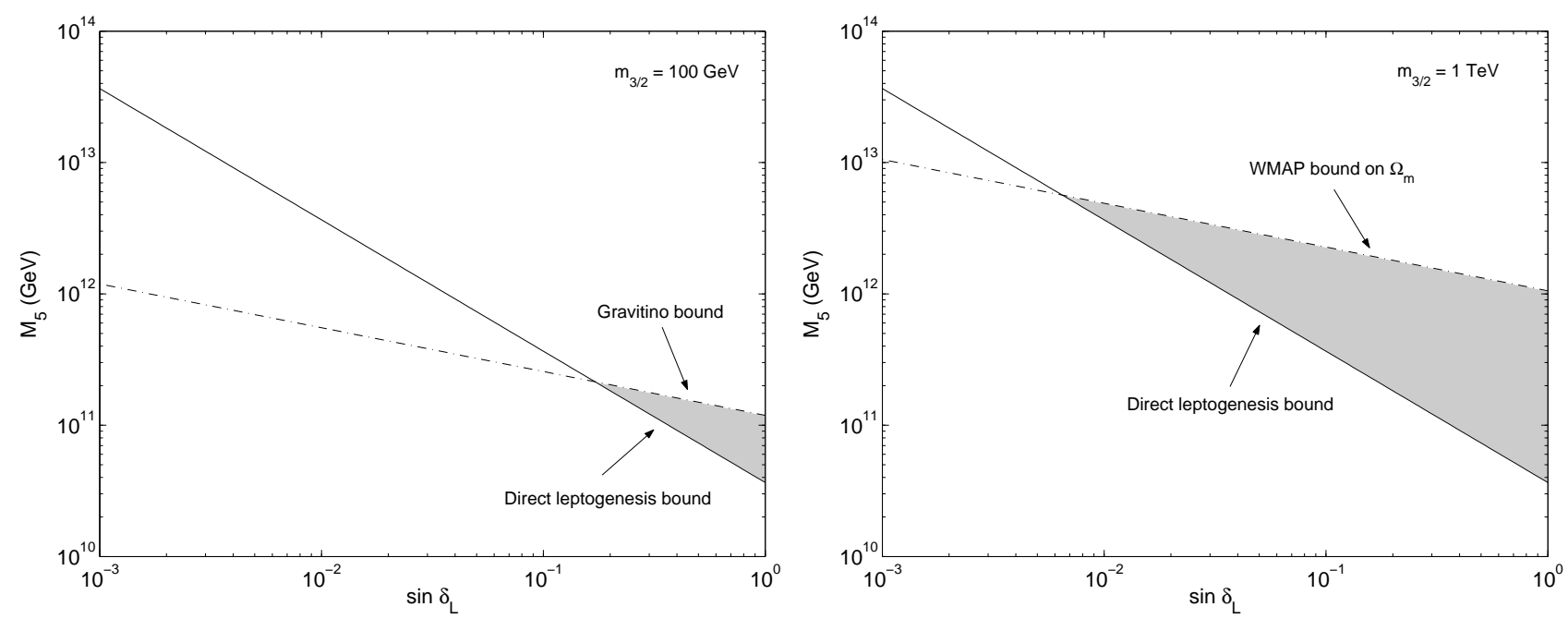

FIG. 1: The 5D Planck mass $M_{5}$ as a function of the effective leptogenesis phase $\sin \delta_{L}$ for $m_{3 / 2}=100 \mathrm{GeV}$ and $m_{3 / 2}=1 \mathrm{TeV}$. The shaded area corresponds to the allowed region, taking into account the BBN constraints on gravitino production, the WMAP bounds on $\eta_{B}$ and $\Omega_{m}$, and assuming direct leptogenesis from sneutrino decays.
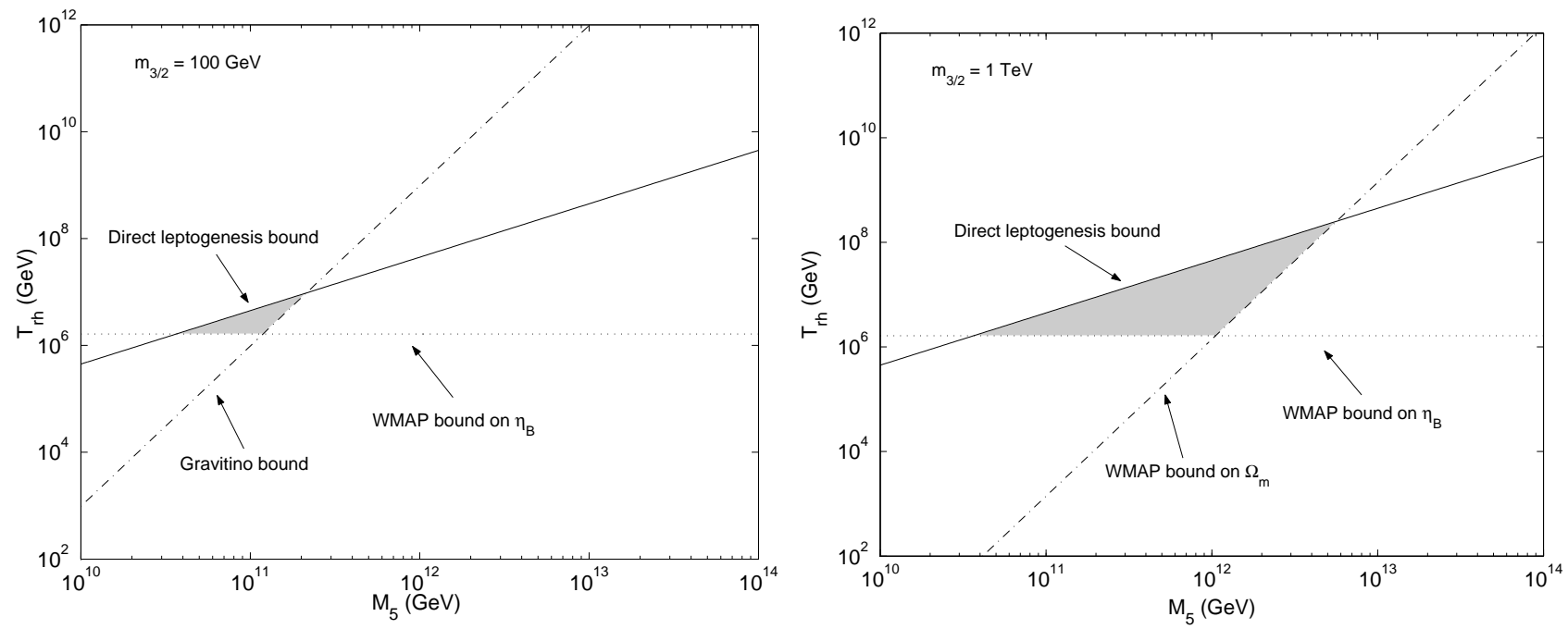

FIG. 2: The reheating temperature $T_{r h}$ as a function of $M_{5}$ for $m_{3 / 2}=100 \mathrm{GeV}$ and $m_{3 / 2}=1$ TeV as derived from the BBN gravitino constraints, direct leptogenesis and the WMAP bounds on $\eta_{B}$ and $\Omega_{m}$. The shaded area corresponds to the region compatible with the above bounds.

(dot-dashed lines) is obtained from Eqs. (45) and (50). The shaded area is the allowed region, which is clearly bigger for larger $m_{3 / 2}$. From this figure we see that the minimum allowed values for the effective leptogenesis phase are $\sin \delta_{L} \gtrsim 0.17$ and $\sin \delta_{L} \gtrsim 6.4 \times 10^{-3}$, for $m_{3 / 2}=100 \mathrm{GeV}$ and $1 \mathrm{TeV}$, respectively.

In Figure 2 we plot the reheating temperature $T_{r h}$ as a function of $M_{5}$, including the bounds from gravitino production (dot-dashed) (cf. Eqs. (45) and (50)), the direct leptogenesis bound (solid) and the bound from Eq. (37), obtained from the WMAP result for $\eta_{B}$ (dotted). The shaded area corresponds to the allowed region. From these figures we conclude that the allowed range for $M_{5}$ and $T_{r h}$ is

$$
\begin{aligned}
& 3.6 \times 10^{10} \mathrm{GeV} \lesssim M_{5} \lesssim 2.1 \times 10^{11} \mathrm{GeV}, \\
& 1.6 \times 10^{6} \mathrm{GeV} \lesssim T_{r h} \lesssim 9.6 \times 10^{6} \mathrm{GeV},
\end{aligned}
$$

if $m_{3 / 2}=100 \mathrm{GeV}$, while for $m_{3 / 2}=1 \mathrm{TeV}$ we find

$$
\begin{aligned}
& 3.6 \times 10^{10} \mathrm{GeV} \lesssim M_{5} \lesssim 5.7 \times 10^{12} \mathrm{GeV}, \\
& 1.6 \times 10^{6} \mathrm{GeV} \lesssim T_{r h} \lesssim 2.6 \times 10^{8} \mathrm{GeV} .
\end{aligned}
$$

Finally, the bounds on the lightest right-handed Majorana neutrino mass, $M_{1}$, are the same as the ones on the reheating temperature.

Braneworld cosmology is a rich subject. During the past few years there has been renewed activity and inter- 
est in this domain. Modifications to the expansion rate of the universe, as is typically the case in braneworld scenarios, can have profound implications for the processes that took place in the early universe. In this paper, we have considered the possibility that two of these phenomena, namely, chaotic inflation and the generation of the baryon asymmetry of the universe through leptogenesis, occurred during the nonconventional era in the brane. We have studied a minimal supersymmetric seesaw scenario where the lightest singlet sneutrino field not only plays the role of the inflaton but also produces a lepton asymmetry through its direct decays. Taking into account the BBN constraints on the gravitino production and the observed baryon asymmetry of the universe, we were able to strongly constrain the fundamental 5D
Planck mass scale, and consequently, the lightest sneutrino mass, as well as the reheating temperature of the universe. The effective leptogenesis phase is also bounded in this framework.

\section{Acknowledgments}

The work of R.G.F and N.M.C.S. was supported by Fundação para a Ciência e a Tecnologia (FCT, Portugal) under the grants SFRH/BPD/1549/2000 and SFRH/BD/4797/2001, respectively. M.C.B. acknowledges the partial support of FCT under the grant POCTI/1999/FIS/36285.
[1] For a review see e.g., D. H. Lyth and A. Riotto, Phys. Rept. 314, 1 (1999) hep-ph/9807278.

[2] C. L. Bennett et al., Astrophys. J. Suppl. 148, 1 (2003) astro-ph/0302207; D. N. Spergel et al., Astrophys. J. Suppl. 148, 175 (2003) astro-ph/0302209.

[3] V. A. Kuzmin, V. A. Rubakov and M. E. Shaposhnikov, Phys. Lett. B 155, 36 (1985).

[4] M. Y. Khlopov and A. D. Linde, Phys. Lett. B 138, 265 (1984); I. V. Falomkin, G. B. Pontecorvo, M. G. Sapozhnikov, M. Y. Khlopov, F. Balestra and G. Piragino, Nuovo Cim. A 79, 193 (1984) [Yad. Fiz. 39, 990 (1984)]; J. R. Ellis, J. E. Kim and D. V. Nanopoulos, Phys. Lett. B 145, 181 (1984); M. Y. Khlopov, Y. L. Levitan, E. V. Sedelnikov and I. M. Sobol, Phys. Atom. Nucl. 57, 1393 (1994) [Yad. Fiz. 57, 1466 (1994)].

[5] R. H. Cyburt, J. R. Ellis, B. D. Fields and K. A. Olive, Phys. Rev. D 67, 103521 (2003) astro-ph/0211258.

[6] H. Murayama, H. Suzuki, T. Yanagida and J. Yokoyama, Phys. Rev. Lett. 70, 1912 (1993).

[7] K. Hamaguchi, H. Murayama and T. Yanagida, Phys. Rev. D 65, 043512 (2002) hep-ph/0109030.

[8] J. R. Ellis, M. Raidal and T. Yanagida, Phys. Lett. B 581, 9 (2004) hep-ph/0303242; G. F. Giudice, A. Notari, M. Raidal, A. Riotto and A. Strumia, hep-ph/0310123

[9] M. Gell-Mann, P. Ramond and R. Slansky, in Supergravity, ed. by P. van Nieuwenhuizen and D. Z. Freedman (North Holland, Amsterdam, 1979), p.315; S. L. Glashow, in Quarks and Leptons, Cargèse, 1979, edited by M. Levy et al. (Plenum, New York, 1980); T. Yanagida, in Proc. of the Workshop on the Unified Theory and Baryon Number in the Universe, ed. by O. Sawada and A. Sugamoto (KEK report 79-18, 1979), p.95, Tsukuba, Japan; ,912; R. N. Mohapatra and G. Senjanovic, Phys. Rev. Lett. 44, 912 (1980).

[10] P. Binetruy, C. Deffayet and D. Langlois, Nucl. Phys. B 565, 269 (2000) hep-th/9905012 ; C. Csaki, M. Graesser, C. F. Kolda and J. Terning, Phys. Lett. B 462, 34 (1999) hep-ph/9906513; J. M. Cline, C. Grojean and G. Servant, Phys. Rev. Lett. 83, 4245 (1999) hep-ph/9906523; T. Shiromizu, K. i. Maeda and M. Sasaki, Phys. Rev. D 62, 024012 (2000) gr-qc/9910076; P. Binetruy, C. Deffayet, U. Ellwanger and D. Langlois, Phys. Lett. B 477,
285 (2000) hep-th/9910219; P. Kraus, JHEP 9912, 011 (1999) hep-th/9910149; D. Ida, JHEP 0009, 014 (2000) gr-qc/9912002; S. Mukohyama, T. Shiromizu and K. i. Maeda, Phys. Rev. D 62, 024028 (2000) [Erratum-ibid. D 63, 029901 (2001)] hep-th/9912287.

[11] L. Randall and R. Sundrum, Phys. Rev. Lett. 83, 4690 (1999) hep-th/9906064.

[12] For other recent studies of baryogenesis in the braneworld context see e.g.: G. R. Dvali and G. Gabadadze, Phys. Lett. B 460, 47 (1999) hep-ph/9904221; A. Masiero, M. Peloso, L. Sorbo and R. Tabbash, Phys. Rev. D 62, 063515 (2000) hep-ph/0003312; R. Allahverdi, K. Enqvist, A. Mazumdar and A. Perez-Lorenzana, Nucl. Phys. B 618, 277 (2001) hep-ph/0108225; A. Mazumdar, Phys. Rev. D 64, 027304 (2001) hep-ph/0007269; A. Mazumdar and A. Perez-Lorenzana, Phys. Rev. D 65, 107301 (2002) hep-ph/0103215; M. BasteroGil, E. J. Copeland, J. Gray, A. Lukas and M. Plumacher, Phys. Rev. D 66, 066005 (2002) hep-th/0201040; T. Matsuda, Phys. Rev. D 65, 103501 (2002) hep-ph/0202209; M. R. Martin, Phys. Rev. D 67, 083503 (2003) hep-ph/0211372; G. L. Alberghi, R. Casadio and A. Tronconi, hep-ph/0310052 A. Mazumdar and A. Perez-Lorenzana, hep-ph/0311106

[13] K. R. Dienes, E. Dudas and T. Gherghetta, Nucl. Phys. B 557, 25 (1999) hep-ph/9811428.

[14] N. Arkani-Hamed, S. Dimopoulos, G. R. Dvali and J. March-Russell, Phys. Rev. D 65, 024032 (2002) hep-ph/9811448.

[15] J. M. Cline, C. Grojean and G. Servant, Phys. Rev. Lett. 83, 4245 (1999) hep-ph/9906523; J. D. Bratt, A. C. Gault, R. J. Scherrer and T. P. Walker, Phys. Lett. B 546, 19 (2002) astro-ph/0208133.

[16] R. Maartens, D. Wands, B. A. Bassett and I. Heard, Phys. Rev. D 62, 041301 (2000) hep-ph/9912464.

[17] D. Langlois, R. Maartens and D. Wands, Phys. Lett. B 489, 259 (2000) hep-th/0006007; G. Huey and J. E. Lidsey, Phys. Lett. B 514, 217 (2001) astro-ph/0104006.

[18] H. V. Peiris et al., Astrophys. J. Suppl. 148, 213 (2003) astro-ph/0302225.

[19] A. R. Liddle and A. J. Smith, Phys. Rev. D 68, 061301 (2003) astro-ph/0307017.

[20] S. Dodelson and L. Hui, Phys. Rev. Lett. 91, 131301 
(2003) astro-ph/0305113; A. R. Liddle and S. M. Leach, Phys. Rev. D 68, 103503 (2003) astro-ph/0305263.

[21] B. Wang and E. Abdalla, arXiv:hep-th/0308145

[22] S. Davidson and A. Ibarra, Phys. Lett. B 535, 25 (2002) hep-ph/0202239.

[23] T. Hambye, Y. Lin, A. Notari, M. Papucci and A. Strumia, hep-ph/0312203
[24] S. Y. Khlebnikov and M. E. Shaposhnikov, Nucl. Phys. B 308 (1988) 885; J. A. Harvey and M. S. Turner, Phys. Rev. D 42, 3344 (1990).

[25] M. Bolz, A. Brandenburg and W. Buchmuller, Nucl. Phys. B 606, 518 (2001) hep-ph/0012052. 